\title{
The effect of a low carbohydrate high fat diet on apolipoproteins and cardiovascular risk
}

\author{
Deaglan McCullough ${ }^{1,2}$, Tanja Harrison ${ }^{1}$, Katie Lane ${ }^{1}$, Lynne Boddy ${ }^{2}$, \\ Farzad Amirabdollahian ${ }^{3}$, Michael Schmidt ${ }^{4}$, Kevin Enright ${ }^{1}$, Claire Stewart ${ }^{2}$ and Ian Davies ${ }^{1}$ \\ ${ }^{1}$ School of Sport Studies, Leisure and Nutrition, Liverpool John Moores University, Liverpool, United Kingdom, \\ ${ }^{2}$ School of Sports and Exercise Sciences, Liverpool John Moores University, Liverpool, United Kingdom, \\ ${ }^{3}$ School of Health Sciences, Liverpool Hope University, Liverpool, United Kingdom and \\ ${ }^{4}$ Advanced Pattern Analysis and Countermeasures Group, Research Innovation Centre, Fort Collins, USA
}

\begin{abstract}
Apolipoproteins (apo) regulate lipoprotein characteristics and lipid metabolism. ApoC-III is a regulator of triglyceride-rich lipoprotein (TRL) metabolism and apolipoproteins are important biomarkers for cardiovascular disease (CVD) risk prediction. A low carbohydrate high fat (LCHF) diet improves cardiometabolic risk, especially via reduction of TRL. However, few studies have compared a LCHF vs. a high carbohydrate (HC), lower fat diet under ad libitum conditions on apoC-III levels. The objectives of this investigation were to measure the effect of a LCHF vs. a HC diet on apoC-III, apoA1, apoB and apoB/apoA1 in 16 healthy Caucasian adults aged 19-64. Ethical approval: Liverpool John Moores University Research Ethics Committee (16/ELS/029); registered with ClinicalTrials. gov (Ref. NCT03257085). Participants randomly assigned to a HC diet (UK Eatwell guidelines; $\geq 50 \%$ of energy from carbohydrates) $(\mathrm{n}=8)$, or a LCHF diet (consume $<50 \mathrm{~g} /$ day of carbohydrates) $(\mathrm{n}=8)$ provided plasma samples at 0,4 and 8 weeks. ApoA1 and apoB were analysed by an automated chemistry analyser (Daytona, Randox Laboratories Ltd, UK). ApoC-III was analysed via ELISA (Thermo Fisher Ltd, USA). Factorial $2 \times 3$ ANOVA and ANCOVA (IBM SPSS $25^{\circledR}$ ) were undertaken to investigate significant differences and to control for variables influenced by baseline measures and visceral adipose tissue (VAT). Results show 0 , 4, and 8 weeks respectively: ApoC-III (LCHF: 19.12 $\pm 9.14,16.05 \pm 7.95,15.11 \pm 3.17 \mathrm{mg} / \mathrm{dl} ; \mathrm{HC}: 22.13 \pm 8.38,28.22 \pm 13.85,22.22 \pm 7.7 \mathrm{mg} / \mathrm{dl}$ ) showed no significant $(P=0.319)$ change. No significant $(P=0.23)$ change was also observed in ApoB (LCHF: 107.25 \pm 20.35 , $111.38 \pm 24.81,111.43 \pm 19.93 \mathrm{mg} / \mathrm{dl}$; HC: $94.38 \pm 20.79,105.00 \pm 20.13,99.00 \pm 29.09 \mathrm{mg} / \mathrm{dl})$. Similarly apoA1 (LCHF: $158.71 \pm$ $14.27,166.50 \pm 23.09,173.00 \pm 29.42 \mathrm{mg} / \mathrm{dl}$; HC: $164.71 \pm 30.25,172.50 \pm 29.44,174.00 \pm 32.83 \mathrm{mg} / \mathrm{dl})$ showed no significant change $(P=0.76)$. This resulted in a relatively unchanged apoB/A1 throughout the study in both diets $(P=0.30)$. No significant $(P>0.05)$ differences were found after 4 weeks or between groups also. ANCOVA revealed a trend $(P=0.06)$ in apoC-III for a difference between groups (LCHF: $\Delta-6.6 \mathrm{mg} / \mathrm{dl}$ vs. HC: $\Delta 1.2 \mathrm{mg} / \mathrm{dl})$ after 8 weeks but no significant $(P>0.05)$ changes in other apolipoproteins were detected. These preliminary data reveal that a LCHF diet does not improve the apolipoprotein profile; however, when accounting for other metabolic risk factors (i.e. VAT) there was a trend towards lowering apoC-III levels $(P=0.06)$. Modulation of apoC-III may lead to improved lipid metabolism, but higher-powered studies are warranted before any improvement on CVD risk can be inferred.
\end{abstract}

\section{Conflict of Interest}

There is no conflict of interest. 